\title{
Simulation of Schottky Barrier MOSFET's with a Coupled Quantum Injection/Monte Carlo Technique
}

\author{
Brian Winstead and Umberto Ravaioli, Senior Member, IEEE
}

\begin{abstract}
A full-band Monte Carlo device simulator has been used to analyze the performance of sub-0.1 $\mu \mathrm{m}$ Schottky barrier MOSFET's. In these devices, the source and drain contacts are realized with metal silicide, and the injection of carriers is controlled by gate voltage modulation of tunneling through the source barrier. A simple model treating the silicide regions as metals, coupled with an Airy function approach for tunneling through the barrier, provides injecting boundary conditions for the Monte Carlo procedure. Simulations were carried out considering a p-channel device with $270 \AA$ gate length for which measurements are available. Our results show that in these structures there is not a strong interaction with the oxide interface as in conventional MOS devices and carriers are injected at fairly wide angles from the source into the bulk of the device. The Monte Carlo simulations not only give good agreement with current-voltage $(I-V)$ curves, but also easily reproduce the subthreshold behavior since all the computational power is devoted to simulation of channel particles. The simulations also clarify why these structures exhibit a large amount of leakage in subthreshold regime, due to both thermionic and tunneling emission. Computational experiments suggest ways to modify the doping profile to reduce to some extent the leakage.
\end{abstract}

Index Terms-Monte Carlo methods, MOSFET's, nanotechnology, Schottky barriers, simulation.

\section{INTRODUCTION}

A GGRESSIVE scaling of silicon integrated devices in the deep-submicron range presents considerable challenges to device designers. Device performance must be preserved as much as possible, when going from one generation to the next, while the design must be manufacturable and cost-effective. As the milestone of $0.1 \mu \mathrm{m}$ gate length is approached for commercial metal oxide semiconductor field effect transistors (MOSFET's), alternative device structures are being considered that might allow the continuation of scaling trends when the physical limits of conventional MOSFET's are eventually reached.

Among the structures under consideration is the Schottky barrier (SB) MOSFET, schematically shown in Fig. 1. In this device, the implanted/diffused highly doped regions for source and drain contacts are replaced by silicide regions, which essentially behave like metals. The structure is quite simple to manufacture

Manuscript received July 20, 1999; revised January 18, 2000. This work was supported in part by the Semiconductor Research Corporation under Contracts 98-SJ-406 and 99-NJ-726. The review of this paper was arranged by Editor A H. Marshak.

The authors are with the Beckman Institute and the Department of Electrical and Computer Engineering, University of Illinois at Urbana-Champaign, Urbana, IL 61801 USA (e-mail: ravaioli@uiuc.edu).

Publisher Item Identifier S 0018-9383(00)04258-1. with a self-aligned process. Because of the sharp silicon/silicide interface, extremely short channels can be achieved. Several experimental structures have been fabricated using a conventional MOSFET configuration [1], [2] as well as silicon-on-insulator (SOI) technology [3].

Despite the topological similarities, the SB MOSFET and the conventional MOSFET have very different physical behavior. Carrier flow from the source contact to the conduction channel takes place mainly by tunneling through the Schottky barrier at the silicon/silicide interface. The width of the barrier is spatially modulated by the gate voltage, and conduction occurs when the barrier is sufficiently thin. Figs. 2 and 3 show a typical potential profile along the channel at a location close to the oxide interface, for two values of the gate bias. When the gate voltage is small (Fig. 2) the barrier between source and channel is sufficiently wide so that relatively little tunneling takes place. A certain amount of leakage current due to thermionic emission from the metal may be present, originating from the tail of the quasiequilibrium distribution in the silicide. For higher gate voltage (Fig. 3) the Schottky barrier just below the oxide interface may become sufficiently narrow to allow tunneling. A Schottky barrier is also present at the drain-channel interface, where it could actually impede the flow of carriers at very low drain voltage.

The silicide material should be chosen so that a reasonable compromise can be reached between junction isolation properties in equilibrium and injection capability when a gate bias is applied. At room temperature, it has been suggested that a Schottky barrier in the range $0.2-0.25 \mathrm{eV}$ is optimal. Of the known silicides, PtSi can be used for p-channel [2] while $\mathrm{ErSi}_{2}$ is suitable for $\mathrm{n}$-channel devices [3].

Although the main injection mechanism is quantum tunneling through the source barrier, the transport in the channel should retain semi-classical features at room temperature even for very short channels, since the scattering phenomena in silicon are sufficiently strong to break quantum coherence. Therefore, we adopted a simulation scheme that couples quantum mechanical injection, through the contact barriers, with a full band Monte Carlo simulation approach for the particle transport. This model has been applied to simulate ultra-small Schottky-barrier MOSFET's and results have been compared with available measurements for $\mathrm{p}$-channel devices with $270 \AA$ A channel length. The simulations show that transport in Schottky-barrier devices does not follow the typical patterns found in conventional MOSFET's, and provide an understanding of the physical reasons for behavior and limitations, specific to this proposed technology. 


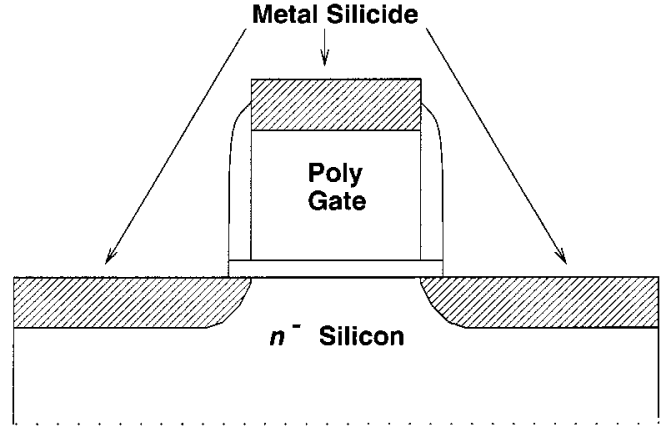

Fig. 1. Schematic structure of a Schottky barrier MOSFET. Only light doping exists in the channel, and the source and drain are metal silicide.

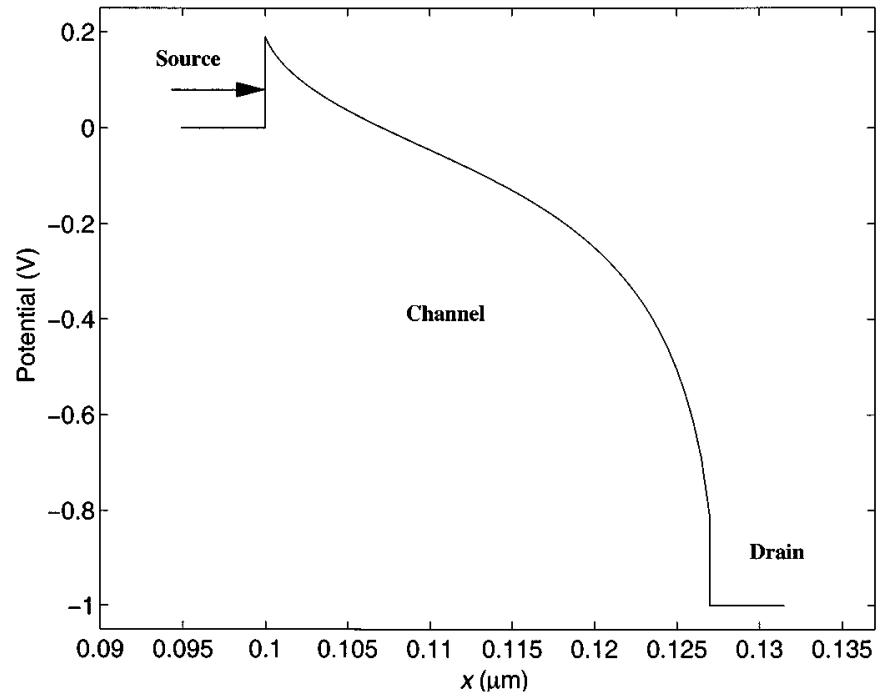

Fig. 2. Potential energy profile along the interface of a Schottky barrier MOSFET under subthreshold conditions. The thickness of the barrier significantly reduces the tunneling current.

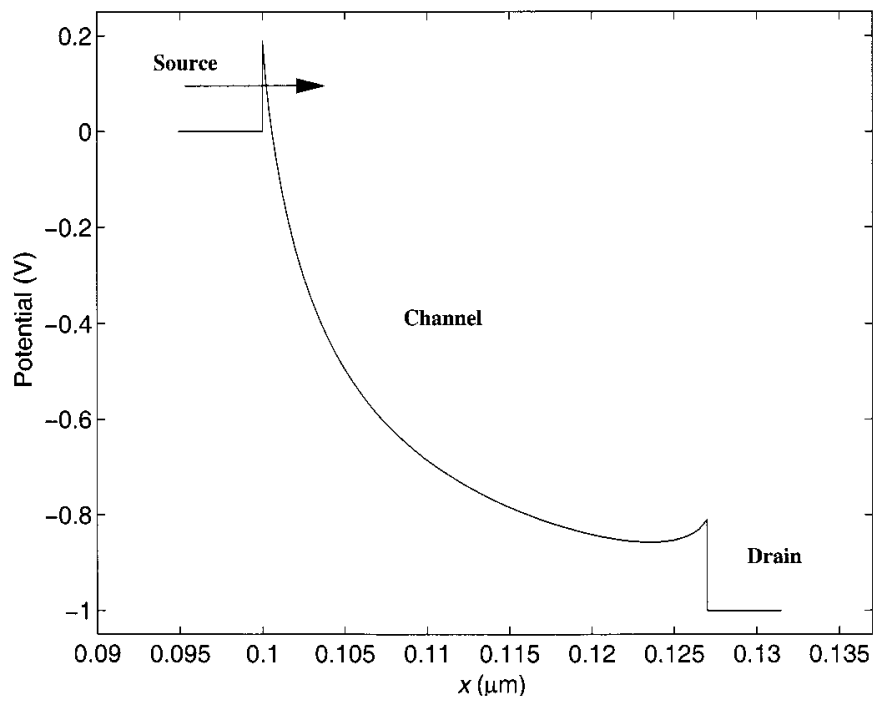

Fig. 3. Potential energy profile along the interface of a Schottky barrier MOSFET under high-field conditions. The barrier becomes transparent to current flow as the barrier becomes thin enough for quantum mechanical tunneling.

\section{SimULATION APPROACH}

The simulated structure is idealized in a number of ways in order to arrive at a computationally manageable model. The silicide contacts are not easy to characterize in terms of precise geometry and material bandstructure, and even the nature of the Schottky barrier may be influenced greatly by device processing. Since the Monte Carlo technique for transport in the bulk of the device can be considered to be quite reliable, for our purposes the most important concern is to capture the essential behavior of the contacts, modeled as reservoirs separated from the silicon layer by a barrier. The silicide contacts are treated as simple metal regions that provide suitable boundary conditions for quantum mechanical tunneling and thermionic emission injection at the silicon interfaces. The distribution function for available carriers in the silicide is evaluated under quasiequilibrium assumptions at the operating temperature.

The detailed bandstructure of the silicide is not well known, therefore, we adopt an ideal metal model, based on a simple parabolic band. In this equivalent metal model, the only parameters needed are the position of the Fermi level with respect to the conduction band and the average effective mass. The effective mass $m^{*}$ is treated as the main tunable parameter in the overall procedure, and it is calibrated against available experimental data for current injection. For our calculations we used an effective mass value of $0.35 m_{0}$, but one should keep in mind that this value must be interpreted as an empirical average and it does not have a rigorous physical meaning.

The injection between contacts and device channel is handled by using transmission probabilities, which are solutions of the Schrödinger equation along paths perpendicular to the silicide/silicon interface. The potential along these paths is taken from the solution of the Poisson equation at each self-consistent step of the Monte Carlo procedure, to recalculate the transmission probabilities as the simulation progresses in time.

The transmission probability is calculated with a standard Airy function approach [4] based on the one-dimensional (1-D) Schrödinger equation on the propagation path

$$
\begin{gathered}
-\frac{\hbar^{2}}{2} \frac{\partial}{\partial z} \frac{1}{m^{*}(z)} \frac{\partial \psi(z)}{\partial z}+V(z) \psi(z)=E_{\text {inc }} \psi(z) \\
E_{\text {inc }}=E_{\text {total }}-\frac{\hbar^{2}}{2 m^{*}(z)} k_{\|}^{2}
\end{gathered}
$$

where $E_{\text {inc }}$ is the kinetic energy in the direction of propagation and $k_{\|}$is the transverse momentum. A transfer-matrix approach is applied, where the potential is interpolated linearly between the grid points on which the Poisson equation is solved in the Monte Carlo region. The solution of (1) in each linear region is a well-known analytical form consisting of Airy functions. The unique solution is then simply calculated through a sequence of $2 \times 2$ matrix multiplications which are equivalent to applying the boundary conditions of continuity of wave function and its derivative at each grid point. We decided to use the Airy function approach, instead of the simpler and computationally cheaper WKB approach, because the WKB model neglects quantum mechanical reflection for the thermionic emission and is typically inaccurate for tunneling near the top of the poten- 
tial barrier. Direct solution of the Schrödinger equation, as implemented here with the Airy function formalism, has also the advantage of treating seamlessly thermionic emission and tunneling injection over the energy range.

For simplicity, we implemented contact regions with a rectangular cross section, taking care of smoothing out the internal corners, to avoid any unphysical peak in the electric field that would correspond to a spurious surge in the carrier injection. The overall geometry of the contacts is chosen as a best compromise to represent the top active layer of the experimental structure presented in [1].

To compute the current injected by the silicide contacts, a transmission coefficient $T\left(E_{i n c}, k_{\|}\right)$is defined as the ratio of the transmitted and incident probability current densities

$$
T\left(E_{\text {inc }}, k_{\|}\right)=\frac{J_{\text {transmitted }}}{J_{\text {incident }}}=\frac{\left|\psi_{2}\right|^{2} k_{2}}{m_{2}^{*}} \cdot \frac{m_{1}^{*}}{\left|\psi_{1}\right|^{2} k_{1}} .
$$

Here, the subscripts 1 and 2 correspond to silicide and silicon, respectively, and $|\psi|^{2}$ represents the wave function probability density for the carriers. At each iteration, a table of transmission probabilities is generated for each mesh location along the contact interface. Once the quasiequilibrium distribution in the silicide is determined in terms of incident energy, the injected current density is obtained by integrating the product between carrier distribution and transmission probability. In practical applications, the influence of the transverse momentum in the transmission probability tends to be actually small. To deal with injection into the contacts for particles treated by Monte Carlo procedure in the silicon region that reach the interface barrier, we separately evaluate the transmission probability for each particle and a random number technique is used to decide whether the particle is absorbed or not.

We have implemented a Monte Carlo simulator for both $\mathrm{n}$ and p-channel devices, but we mainly consider here a p-channel device with PtSi contacts because of the availability of measurements. We assume a Schottky barrier height of $0.2 \mathrm{eV}$ for the $\mathrm{PtSi} / \mathrm{Si}$ interface. Barrier lowering due to the image force is included in the potential used for the quantum mechanical calculations. Holes are injected in the channel by considering the detail of the Si valence band. Injection is proportional to the density of states of the different valence band branches. However, transfer involves mainly the heavy and light hole bands since the spin-orbit split band has a bigger barrier.

The Monte Carlo model used for the calculations is similar to the one described in [5]. The numerical band structure was included for the valence band, by using a local pseudo-potential band solution [6]. The Poisson equation is solved every time step (on the order of $1 \mathrm{fs}$ ) to achieve self-consistency. A light n-type doping is assumed for the substrate and the Poisson equation is solved in nonlinear form, using a standard conjugate gradient approach, in order to obtain the correct depletion and band bending in the bulk of the device. Typically, up to 10000 holes were included in the calculations. It should be noted that Monte Carlo simulation of devices usually require simulation of large number of particles to account for the contacts. Since the behavior of silicide contacts can be entirely represented by injection boundary conditions, dependent on a known quasiequilibrium distribution, the complete ensemble of simulated particles

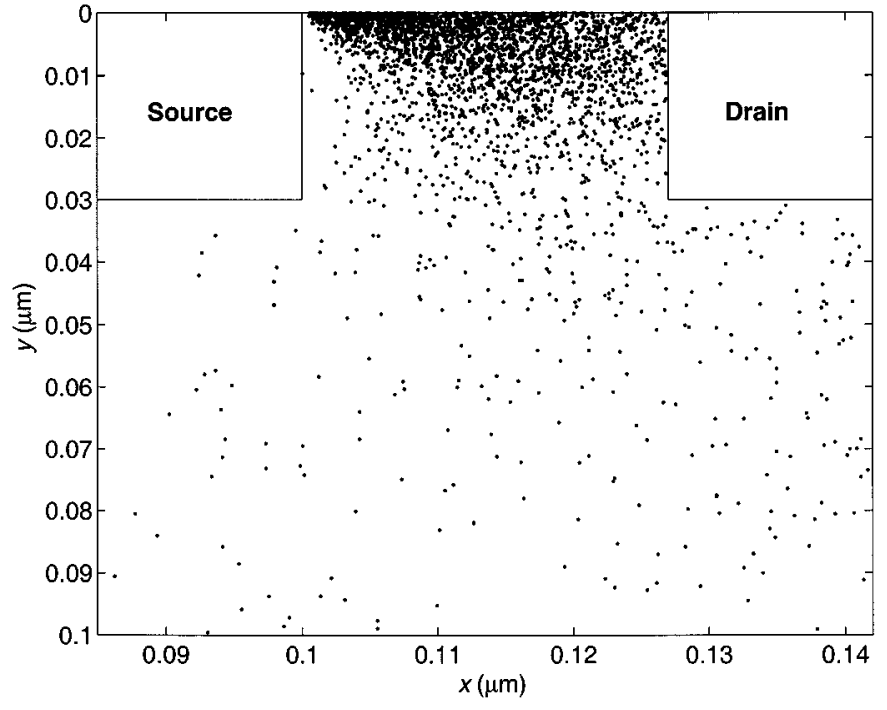

Fig. 4. Typical particle snapshot in time for the Monte Carlo simulation of a $270-\AA$ p-channel Schottky barrier MOSFET in saturation bias. Approximately $90 \%$ of the current is injected within $50 \mathrm{~A}$ of the $\mathrm{Si}_{-}-\mathrm{SiO}_{2}$ interface.

is applied to transport in the channel. This greatly improves the performance and resolution of the method, and allows one to easily probe the subthreshold regime, conditions which would normally require a prohibitive number of particles for conventional MOSFET's, in order to have a significant number of particles in the channel.

\section{Simulation Results}

We have simulated a p-channel silicide MOSFET with 270 $\AA$ channel length and $19 \AA$ À oxide thickness, with $\mathrm{n}^{+}$-polysilicon gate, similar to the experimental device presented in [1]. The substrate is lightly doped with a concentration $N_{D}=10^{15}$ $\mathrm{cm}^{-3}$. The depth of the silicide junction is $400 \AA$ A. Fig. 4 shows a snapshot of the Monte Carlo simulation, with the distribution of holes in the device, for drain bias $V_{d}=-1.0 \mathrm{~V}$ and gate bias $V_{g}=-2.1 \mathrm{~V}$. This picture offers clear indications of how the operation of SB MOSFET's differs from conventional MOS devices. Most of the injection into the channel takes place through a narrow region of the source-channel junction, where the gate bias has the effect of narrowing the Schottky barrier. The stream of carriers enters the channel like a spray with a fairly broad angle. While the integrated charge density at different $x$-coordinates along the channel remains relatively constant, the distribution spreads out into the bulk as the carriers move from source to drain. The carriers are not closely bound to the surface as in a conventional MOSFET because of low doping in the channel. In addition, high fields are located near the source instead of near the drain. Carriers injected from the source quickly obtain a high velocity enabling them to avoid significant surface interaction. An important consequence of this fact is that interface roughness scattering seems to have a negligible influence on device operation. Simulations with or without the inclusion of the interface scattering mechanism yield practically the same result.

The measured $I-V$ curves from Fig. 3 of [1] are reproduced in Fig. 5 along with the corresponding simulated results, for the range of gate voltages from -1.7 to $-2.3 \mathrm{~V}$. There is good 


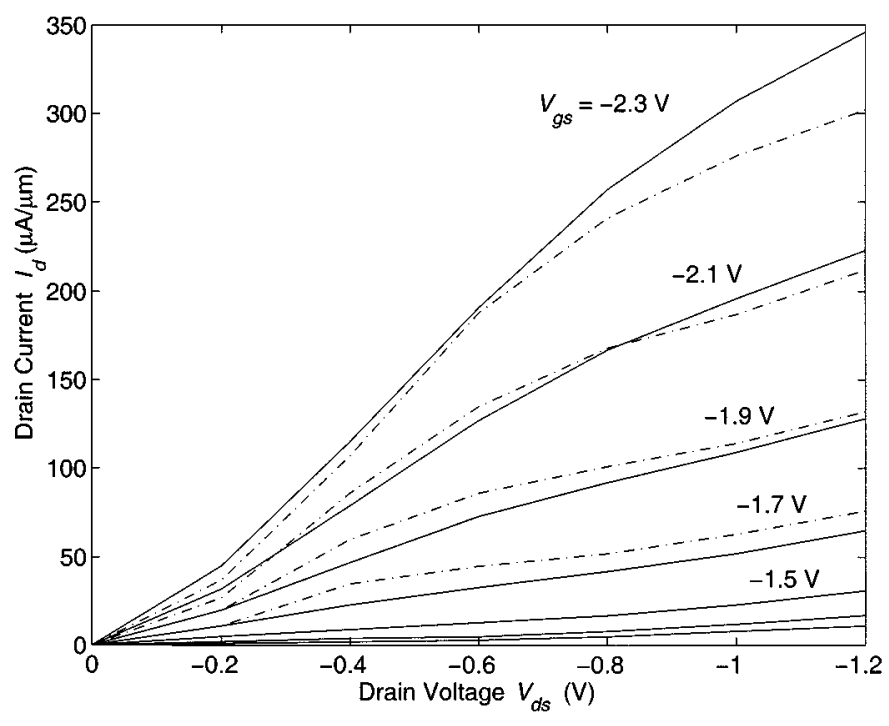

Fig. 5. $I-V$ characteristics of a $270 \AA$ A -channel Schottky barrier MOSFET. The oxide thickness is $19 \AA$ A. The solid lines are experimental results from [1] and the dash-dot lines are from Monte Carlo simulation.

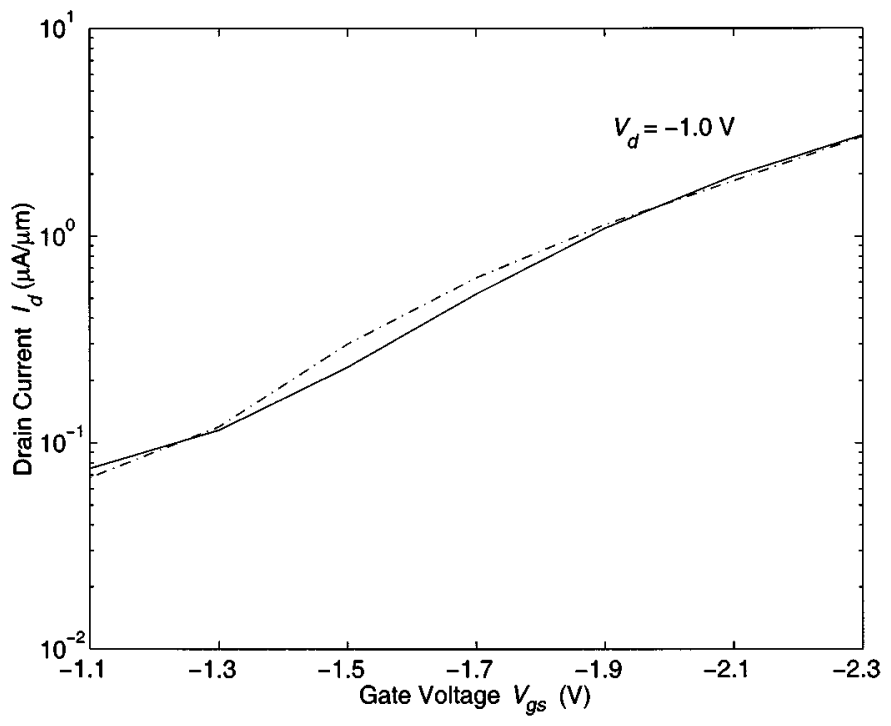

Fig. 6. Subthreshold characteristics of the same device in Fig. 5. The solid line is an experimental result from [1] and the dash-dot line is from Monte Carlo simulation.

agreement overall. One of the most noticeable features is the sub-linear behavior of the current at very small drain voltages. This effect is due to the Schottky barrier at the drain, impeding current flow until the drain bias is sufficiently high. From the point of view of circuit design, this is not a desirable behavior. Simulation of the subthreshold current shows a remarkable agreement with the experimental data, as shown in Fig. 6. As mentioned earlier, Monte Carlo results for subthreshold conditions are readily obtained, since all the simulated particles are used to sample conduction in the channel, and the close agreement indicates that the coupled quantum-injection/Monte Carlo model should be valid for silicon devices with such short channel lengths as considered here. However, the subthreshold results also point out the major problem of the analyzed device structure. While the drive current is reasonable for practical

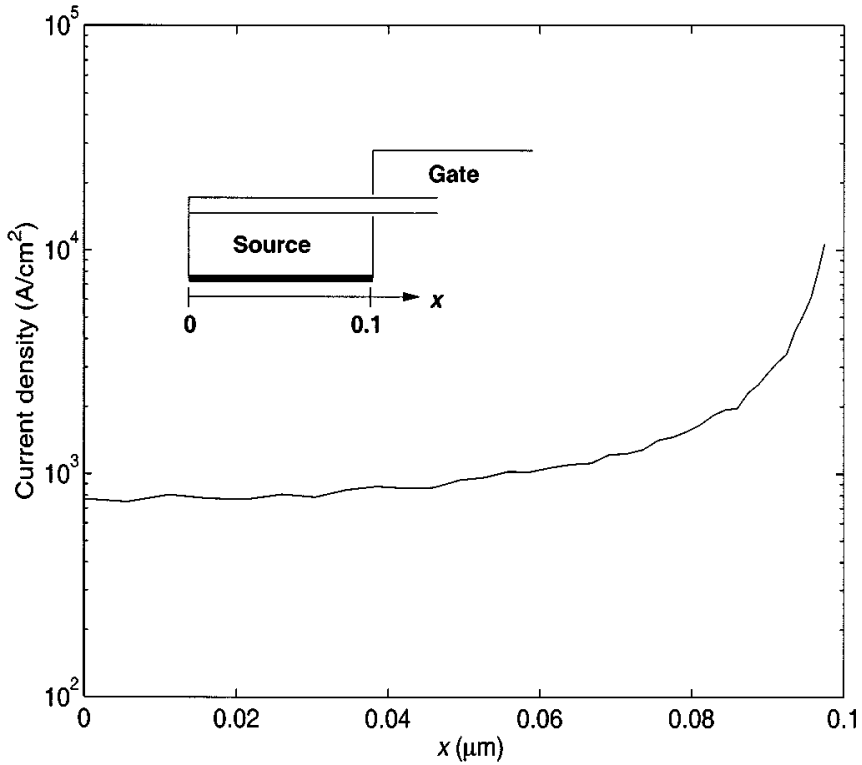

Fig. 7. Current density along the substrate-facing side of the source in subthreshold bias, $V_{d}=-1.0 \mathrm{~V}, V_{g}=0.0 \mathrm{~V}$. Leakage is primarily due to thermionic emission over the fixed silicide-silicon barrier.

integrated circuit applications, the drain currents in on and off conditions differ by less than two orders of magnitude, which is well below what is normally considered adequate for a standard MOSFET (five to six orders of magnitude). The high off-current is indicative of leakage at the source, and simulations are valuable for a detailed analysis. There is one contribution to the leakage current due to thermionic emission for energies above the nominal barrier, which occurs along the entire perimeter of the source and is essentially independent of bias. A second and equally significant component of the leakage is due to tunneling and to additional thermionic emission, both related to the barrier lowering effects of the drain-source potential and the gate-source workfunction. The effect of the drain-induced barrier lowering (DIBL), is illustrated in Figs. 7 and 8, which show the current density flowing out of the source sides facing the substrate and facing the channel, in conditions of subthreshold bias. The side facing the substrate primarily produces thermionic leakage over the fixed barrier. The drain bias affects the leakage current only to the extent that it sweeps away carriers which cross the barrier, and in this way the current is mainly flat and starts rising only in the portion closest to the drain. In contrast, the source side facing the channel is more directly affected by barrier lowering and produces leakage current density which is more than one order of magnitude larger. The barrier lowering is in fact so pronounced in the $270 \AA$ device that tunneling emission slightly exceeds thermal emission over the reduced barrier. Close to the oxide interface there is a fixed amount of barrier lowering approximately independent of the drain bias, because of the proximity to source and gate electrodes. Although held at zero bias, there is a field due to the electrochemical potential difference between gate (polysilicon) and source (silicide), which is about the same as the Schottky barrier. The leakage current drops off slightly moving away from the oxide interface, but then it rises again because the barrier lowering becomes 


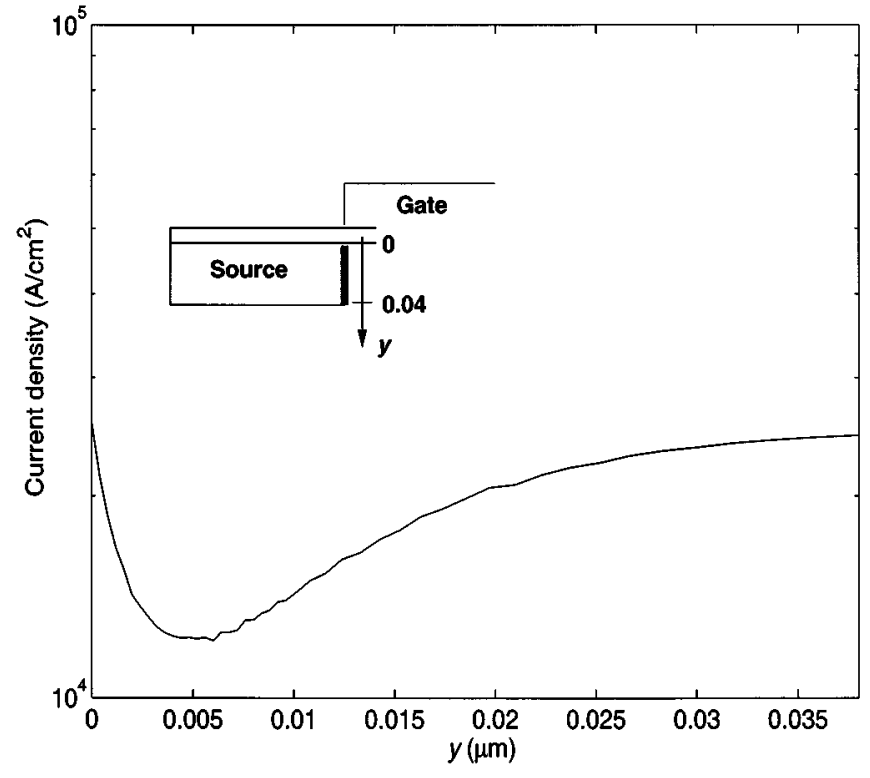

Fig. 8. Current density along the channel-facing side of the source in subthreshold bias, $V_{d}=-1.0 \mathrm{~V}, V_{g}=0.0 \mathrm{~V}$. Leakage current density is more than one order of magnitude larger than on the substrate-facing side. This is due to drain-induced barrier lowering through the mechanism of image force.

dominated by the DIBL effect. DIBL is a short-channel effect, exacerbated by scaling. However, both thermal leakage over the barrier and leakage due the gate-source workfunction are independent of channel length. For this reason, long-channel SB MOSFET's still produce a significant amount of leakage relative to conventional devices.

A natural way to improve the subthreshold behavior is to augment the Schottky barrier at the source by adding doping. This should be done in a way that does not appreciably affect the current drive. The Monte Carlo simulations introduced above have indicated that about $90 \%$ of the source on-current is injected through the first $50 \AA$ below the $\mathrm{Si}_{-} \mathrm{SiO}_{2}$ interface. Additional doping to increase the barrier in this region would have a severe impact on drive, but doping could be introduced in the rest of the device as needed. As illustrated in Fig. 8, leakage only varies slightly along the source side facing the channel. Thus, we expect that the leakage current could be reduced in the most optimistic case to be $I \approx(50 \AA /$ junction depth $) I_{0}$, where $I_{0}$ represents the leakage current before doping modifications. A Monte Carlo simulation was performed, showing that this estimate is reasonable. For a similar device with $270-\AA$ channel and $400 \AA$ junction depth at the source, we added a simple doping profile uniform along the $x$-direction and Gaussian along the $y$-direction. The density peak was chosen to be $10^{18} \mathrm{~cm}^{-3}$ at the source junction depth, with $\sigma \approx 200 \AA$. The simulations show that, in exchange for a $15 \%$ reduction in drive current, the on/off ratio can be increased to 150 from the value of 25 inferred from Fig. 6. Although this is a good improvement, the overall leakage behavior still falls short of what is normally expected of standard MOSFET's. In the case of SOI technology, the leakage would be reduced in a similar way, due to the reduction of source contact area in communication with the channel.

Another possible, although technologically problematic, degree of freedom in the design of the SB MOSFET is changing

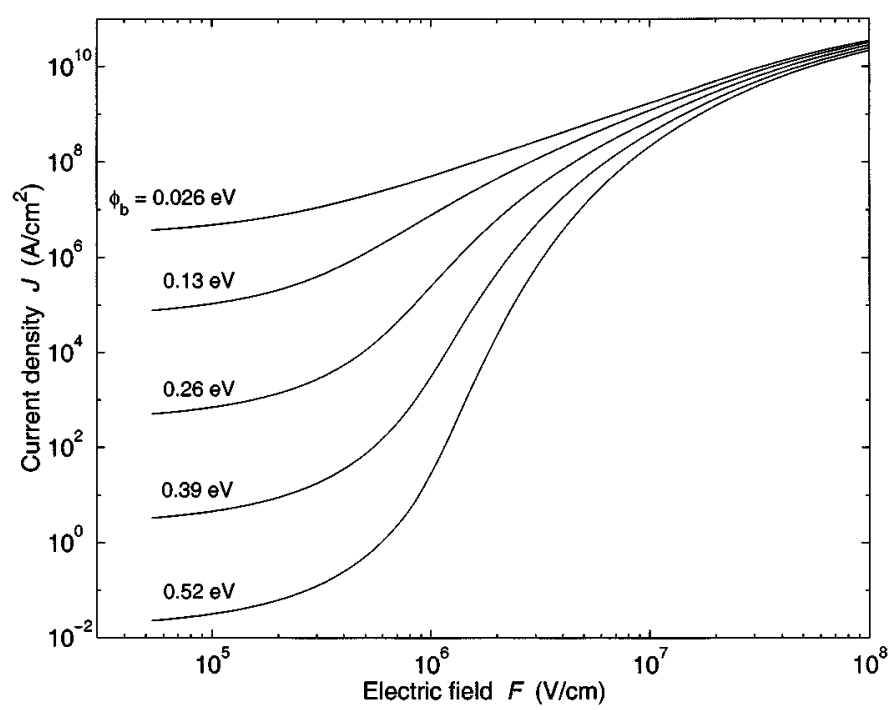

Fig. 9. Comparative behavior of current turn-on simulated by varying the Schottky barrier in an isolated silicide/silicon junction as a function of field characterizing the corresponding triangular barrier.

the source barrier height. For example, we performed Monte Carlo simulations of the same $270-\AA$ device with barrier height increased from $0.2 \mathrm{eV}$ to $0.47 \mathrm{eV}$ obtaining an improvement of the on/off ratio from 25 to $10^{4}$. However, the current drive was reduced by almost a factor of ten. To provide a practical engineering criterion, we examined an isolated Schottky barrier and evaluated the injected current through the corresponding barrier, for different values of the Schottky barrier and a wide range of fields characterizing the triangular potential. The results are shown in Fig. 9. For maximum control by the gate voltage, a high barrier should be used and the Schottky barrier MOSFET should be biased in a regime where the current variations are maximized. However, for practical fields in the $\mathrm{MV} / \mathrm{cm}$ range, the penalty for using a larger barrier is a significant reduction in drive current. These curves can be used as a qualitative guideline to understand the best tradeoff between drive and isolation.

\section{ConClusions}

We have demonstrated a coupled quantum-injection/Monte Carlo simulation procedure that is suitable to study MOSFET devices with silicide contacts. Monte Carlo simulation of such devices is found to be particularly effective both because uncertainty in the $\mathrm{Si} / \mathrm{SiO}_{2}$ interface model is not significant, and because all computational effort is focused in the channel. Results for a $270 \AA$ p-channel device agree well with available measurements, showing that the use of semi-classical transport in the bulk of the silicon device is still reasonable. The simulations provide detailed information to understand the mechanisms of leakage in subthreshold conditions, thus providing a clear physical explanation of the poor isolation behavior. Modification of the device to improve performance can be guided by simulation. For instance, we estimated how much the addition of an optimal doping profile in the channel or the use of SOI would improve isolation. Injection from the first $50 \AA$ of the source below the $\mathrm{Si}_{-} \mathrm{SiO}_{2}$ interface was shown to be the most critical for both drive and leakage current. To obtain significant 
improvement in device performance, the reduction of leakage beneath the channel with either doping or SOI must be accompanied with a mechanism which can reduce leakage without affecting drive in the first $50 \AA$.

\section{ACKNOWLEDGMENT}

The authors would like to thank C. Wang and J. R. Tucker for providing detailed information about their experimental results.

\section{REFERENCES}

[1] C. Wang, J. P. Snyder, and J. R. Tucker, "Sub-40 nm PtSi Schottky source/drain metal-oxide-semiconductor field-effect transistors," Appl. Phys. Lett., vol. 74, pp. 1174-1176, 1999.

[2] J. P. Snyder, C. R. Helms, and Y. Nishi, "Experimental investigation of a PtSi source and drain field emission transistors," Appl. Phys. Lett., vol. 67, pp. 1420-1422, 1995.

[3] M. Nishisaka and T. Asano, "Reduction of the floating-body effect in SOI MOSFET's by using Schottky source/drain contacts," in Ext. Abs. 1997 Int. Conf. Solid-State Devices and Materials, 1997, pp. 160-161.

[4] K. F. Brennan and C. J. Summers, "Theory of resonant tunneling in a variably spaced multiquantum well structure: An Airy function approach," J. Appl. Phys., vol. 61, pp. 614-623, 1987.

[5] A. Duncan, U. Ravaioli, and J. Jakumeit, "Full-band Monte Carlo investigation of hot carrier trends in the scaling of metal-oxide-semiconductor field-effect transistors," IEEE Trans. Electron Devices, vol. 45, pp. 867-876, May 1998.
[6] M. L. Cohen and J. R. Chelikowsky, Electronic Structure and Optical Properties of Semiconductors, 2nd ed. Berlin, Germany: Springer-Verlag, 1989.

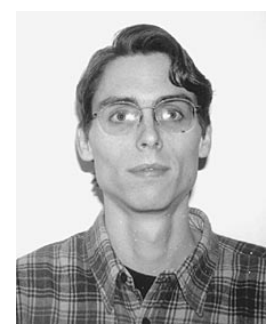

Brian Winstead received the B.S. degree in electrical engineering from the University of Texas, Austin, in 1996, and the M.S. degree in electrical engineering from the University of Illinois, Urbana-Champaign, in 1998.

His primary research interests are Monte Carlo simulation and nanoscale silicon devices.

Umberto Ravaioli (SM'93) received the Laurea degree in electronics engineering in 1980 and the Laurea degree in physics in 1982, both from the University of Bologna, Italy, and the Ph.D. degree in electrical engineering from Arizona State University, Tempe, in 1986.

He joined the Department of Electrical and Computer Engineering, University of Illinois, Urbana-Champaign, in 1986, where he is now a Professor. His research interests are in the areas of Monte Carlo device simulation, nanoelectronics, quantum transport, and supercomputation. 